\title{
Magnetic field effects on electron-hole recombination in disordered organic semiconductors
}

\author{
A. I. Shushin \\ Institute of Chemical Physics, Russian Academy of Sciences, \\ 117977, GSP-1, Kosygin str. 4, Moscow, Russia
}

\begin{abstract}
Characteristic properties of magnetic field effects on spin selective geminate and bulk electron-hole polaron pair (PP) recombination are analyzed in detail within the approach based on the stochastic Liouville equation. Simple expressions for the magnetic field (B) dependence of recombination yield and rate are derived within two models of relative PP motion: free diffusion and diffusion in the presence of well (cage). The spin evolution of PPs is described taking in account the relaxation induced by hyperfine interaction, anisotropic part of the Zeeman interaction induced, as well as $\Delta g$-mechanism. A large variety of the $B$-dependences of the recombination yield $Y(B)$ and rate $K(B)$ is obtained depending on the relative weights of above-mentioned mechanisms. The proposed general method and derived particular formulas are shown to be quite useful for the analysis of recent experimental results.
\end{abstract}

PACS numbers: 73.50.-h, 73.43.Qt, 75.47.-m, 72.25.Dc

\section{INTRODUCTION}

Magnetic field effects (MFEs) on various processes in organic semiconductors are actively studied for many years $\stackrel{1-17}{-17}$ These studies concern different types of MFEs: the magnetic field dependent photoconductivity,$\underline{\underline{3}}$ and photoluminescence, $\stackrel{5,9}{,}$, magnetoelectroluminescence,,$\stackrel{11,13}{,}$ magnetoresistance,,$\frac{4,6-17}{17}$ etc.

The mechanisms of MFEs in these processes are the subjects of hot debates for many years. It is, however, widely accepted, $\stackrel{18-20}{n}$ that a large number of MFEs result from the effect of the magnetic field $(B)$ on the spin selective reactions with participation of paramagnetic particles: polarons $(P)$ and triplet excitons $(T)$. In a large number of them the key stage is the recombination of pairs of electron $(e)$ and hole $(h)$ polarons, i.e. particles with electronic spin $1 / 2,4,8,14,17$ which are called hereafter polaron pairs (PP). The $T-P$ quenching $T-T$ annihilation are also believed be the important spin selective process which can give significant contribution to the observed MFEs in organic semiconductors $18,21-23$

There are some reviews of experimental and theoretical works on MFEs in organic semiconductors $\underline{18-20}$ Recent extensive experimental investigations, however, inspire further theoretical studies of MFEs, $, 4,8,14,17$ Despite evident progress in these studies there are still many problems to be discussed.

In particular, close attention has been attracted to the $e-h \mathrm{PP}$ recombination mechanism of MFEs in disordered semiconductors,$\underline{4,17,18}$ The important problem consists in proper treatment of the effect of polaron migration and disorder of spin dependent interactions, giving rise to the MFEs. The majority of theoretical works are mainly based either on numerical or somewhat simplified analytical description of the spin/space evolution of PPs, $14,17,18$ though fairly high accuracy of above mentioned recent measurements motivates more detailed theoretical investigations, which could allow for obtaining sufficiently ac- curate and rigorous formulas for the MFEs.

In this work we discuss the method of describing specific features of the $\mathrm{PP}$ recombination mechanism of MFEs in disordered organic semiconductors. The method is based on the diffusion approximation for hopping polaron migration, fairly reasonable at long times of MFE formation. The hopping motion is assumed to lead not only to stochastic spatial evolution but also to fluctuations of disordered spin dependent hyperfine interaction (HFI) and anisotropic part of the Zeeman interaction (AZI), resulting from the anisotropy of $g$-factors of polarons. The fluctuating HFI and AZI give rise to spin relaxation, which is described by Bloch-type equations, valid in the realistic limit of hopping rates much larger than these interactions (in frequency units).

The kinetics of MFE generation is determined by the spin/space evolution of PPs, which is described by the $\mathrm{PP}$ spin density matrix. In the above-formulated approximations this matrix satisfies the stochastic Liouville equation (SLE) $\underline{19,24,25}$

With the SLE approach we analyze the properties of MFE for geminate and bulk processes within two models of PP relative motion: free diffusion and diffusion in the presence of a potential well $\stackrel{26,27}{2}$ The PP spin evolution is described taking into consideration the abovementioned HFI and AZI induced relaxation, as well $\Delta g$ mechanism. ${ }^{19}$ In these two models of relative motion simple expressions are derived for the MFE, i.e. for $B$ dependent recombination yield $Y(B)$ and rate $K(B)$ (in geminate and bulk precesses, respectively).

The analysis with obtained formulas reveals different types of $Y(B)$ and $K(B)$ behavior, as $B$ increased, for HFI and AZI induced relaxation mechanisms: decreasing and decreasing, respectively. The combination of these mechanisms is found to result in a large variety of non-monotonic $Y(B)$ and $K(B)$ dependences. The extra contribution of $\Delta g$-mechanism can lead to some additional specific features of MFEs behavior at large magnetic fields $B$ whose specific features appear to depend 
on the mechanism of relative motion of polarons.

In our discussion we also concern some possible applications of obtained expressions to the interpretation of some recent experimental results.

\section{MECHANISM OF POLARON MIGRATION}

The characteristic properties of MFEs on the migration assisted PP recombination are, naturally, essentially determined by the mechanism of polaron migration.

There are a number of models of migration in disorder semiconductors. One of the most popular is the Miller-Abrahams model ${ }^{28,29}$ In this model the rate $w_{i j}$ of hopping from the site $j$ to the site $i$ is written as $w_{i j}=w_{E_{i} E_{j}}=w_{0} e^{-\theta\left(E_{i}-E_{j}\right)\left(E_{i}-E_{j}\right) /\left(k_{B} T\right)}$, where $w_{0}$ is the characteristic rate constant, $\theta(x)$ is the Heaviside step function, $r_{i j}$ is the distance between these sites, and $E_{j}$ and $E_{i}$ are the energies of initial and final states, respectively, assumed to be randomly distributed parameters whose (broad) distribution functions are determined by a number of intra and interpolaron interaction. ${ }^{28,29}$

Even in this relatively simple model the kinetics of the space/time evolution of charge carriers can be obtained only numerically.

It is worth noting, however, that the MFEs are determined by very long times of order of the characteristic time $\tau_{S} \sim 10^{-8} s^{-1}$ of spin evolution of $e$ - and $h$ polarons, 25 which is much longer than the average hopping time $w_{0}^{-1}: \tau_{S} \gg w_{0}^{-1}$ (see Sec. VIII). At times $t \gtrsim \tau_{S} \gg w_{0}^{-1}$ in the wide region of parameters of the model the space/time evolution is fairly reasonably described by the diffusion approximation. One of indications of this fact is a reasonably good accuracy of the Langevin formula for the recombination rate. ${ }^{29}$

In the diffusion approximation the migration kinetics of $\nu$-polaron $(\nu=e, h)$ is described by the time dependent probability distribution function $p_{\nu}\left(\mathbf{r}_{\nu}, t\right)$ in the continuum space $\left\{\mathbf{r}_{\nu}\right\}$, obeying the Smoluchowski equation

$$
\dot{p}_{\nu}=D_{\nu} \nabla_{\mathbf{r}_{\nu}}\left(\nabla_{\mathbf{r}_{\nu}} p_{\nu}+p_{\nu} \nabla_{\mathbf{r}_{\nu}} u_{\nu}\right), \quad(\nu=e, h),
$$

In eq.2.1) $D_{\nu} \sim l_{\nu}^{2} w_{0_{\nu}}$ is the effective diffusion coefficient for $\nu$-polaron, in which $l_{\nu}$ and $w_{0_{\nu}}$ are the characteristic hopping length and hopping rate. Note that eq. (2.1) is actually a selfconsistent equation, which incorporates many particle effects, showing themselves in possible dependence of the diffusion coefficient on the concentration of polarons, as well as concentration and temperature dependence of the effective potential $u\left(\mathbf{r}_{\nu}\right) \cdot 29$

Our further analysis of MFEs will be based on the diffusion approximation (2.1). Nevertheless, some specific features of hopping kinetics at short times, predicted by the Miller-Abrahams model, appear to be important as well for the description of MFEs (see below).

\section{SPIN EVOLUTION}

Quantum (spin) PP evolution plays the key role in the generation of MFEs on $\mathrm{PP}$ recombination. $\mathrm{PP}$ recombination is known to be a spin selective process with the rate depending on the total electron spin of the pair of $e$ - and $h$-polarons: $\mathbf{S}=\mathbf{S}_{e}+\mathbf{S}_{h}$, where $\mathbf{S}_{\nu}$ is the spin of $\nu$-polaron $(\nu=e, h)$. In our discussion we will assume that the rate is non-zero only in the singlet $(S)$ state corresponding to the total PP spin $S=0.18,19$

The spin evolution of $e$ - and $h$-polarons is described by spin density matrices $\rho_{\nu},(\nu=e, h)$, in the two state Hilbert spaces, in which two states $\left|\nu_{ \pm}\right\rangle$correspond to two spin projections onto $z$-axis, taken to be parallel to the vector $\mathbf{B}$ of external magnetic field: $S_{\nu_{z}}\left|\nu_{ \pm}\right\rangle=$ $\pm \frac{1}{2}\left|\nu_{ \pm}\right\rangle$. In general, these matrices can be represented in terms of expansion in bilinear combinations of the states

$$
\left|\nu_{\mu \mu^{\prime}}\right\rangle=\left|\nu_{\mu}\right\rangle\left\langle\nu_{\mu^{\prime}}\right|
$$

It is important to note that $\left|\nu_{\mu \mu^{\prime}}\right\rangle$ can be considered as vectors in some space, called hereafter the Liouville space and denoted as $\left\{\nu_{\mu \mu^{\prime}}\right\}$, in which the conjugated vectors $\left\langle\nu_{\mu^{\prime} \mu}\right|$ are defined by the relation $\left\langle\nu_{\mu_{1} \mu_{1}^{\prime}} \mid \nu_{\mu_{2} \mu_{2}^{\prime}}\right\rangle=$ $\delta_{\mu_{1} \mu_{2}} \delta_{\mu_{1}^{\prime} \mu_{2}^{\prime}}$. Noteworthy is also that this definition implies linear independence of the vectors $\left|\nu_{\mu \mu^{\prime}}\right\rangle$ and $\left|\nu_{\mu^{\prime} \mu}\right\rangle$, i.e. $\left\langle\nu_{\mu^{\prime} \mu} \mid \nu_{\mu \mu^{\prime}}\right\rangle=0$. In $\left\{\nu_{\mu \mu^{\prime}}\right\}$-space, the density matrices $\rho_{\nu}=\sum_{\mu, \mu^{\prime}= \pm} \rho_{\nu_{\mu \mu^{\prime}}}\left|\nu_{\mu}\right\rangle\left\langle\nu_{\mu^{\prime}}\right|$ can be written as a vectors:

$$
\rho_{\nu} \equiv\left|\rho_{\nu}\right\rangle=\sum_{\mu, \mu^{\prime}= \pm} \rho_{\nu_{\mu \mu^{\prime}}}\left|\nu_{\mu \mu^{\prime}}\right\rangle
$$

In addition to vectors, in the Liouville space one can also introduce operators, which will be called superoperators.

The time evolution of the spin density matrix $\rho_{\nu}$ satisfies the Schrödinger equation (hereafter we put $\hbar=1$, i.e. use frequency units for energy parameters)

$$
\dot{\rho}_{\nu}=-i \hat{H}_{\nu} \rho_{\nu}
$$

In this equation $\hat{H}_{\nu}$ the spin Hamiltonian superoperator (operator in the Liouville space) expressed in terms of the Hamiltonian $H_{\nu}$ (in the Hilbert space) by the relation

$$
\hat{H}_{\nu} \rho_{\nu} \equiv \hat{H}_{\nu}\left|\rho_{\nu}\right\rangle=H_{\nu} \rho_{\nu}-\rho_{\nu} H_{\nu}
$$

For matrix elements of $\hat{H}_{\nu}$ we get $\left\langle\nu_{\mu_{1} \mu_{1}^{\prime}}\left|\hat{H}_{\nu}\right| \nu_{\mu_{2} \mu_{2}^{\prime}}\right\rangle=$ $\left\langle\nu_{\mu_{1}}\left|H_{\nu}\right| \nu_{\mu_{2}}\right\rangle \delta_{\mu_{1}^{\prime} \mu_{2}^{\prime}}-\left\langle\nu_{\mu_{2}^{\prime}}\left|H_{\nu}\right| \nu_{\mu_{1}^{\prime}}\right\rangle \delta_{\mu_{1} \mu_{2}}$.

\section{SPIN HAMILTONIAN AND SPIN RELAXATION}

In general the spin Hamiltonian $H_{\nu}\left(\mathbf{r}_{\nu}\right),(\nu=e, h)$, depends on the coordinate $\mathbf{r}_{\nu}$ of $\nu$-polaron, due to inhomogeneity of the medium. This dependence results in fluctuations of $\hat{H}_{\nu}(t) \equiv \hat{H}_{\nu}\left(\mathbf{r}_{\nu}(t)\right)$ caused by hopping motion of $\nu$-polaron, i.e. stochastic changing $\mathbf{r}_{\nu}(t)$. The 
Hamiltonian can conventionally be represented as a sum of the stationary and fluctuating parts:

$$
H_{\nu}\left(\mathbf{r}_{\nu}(t)\right)=\bar{H}_{Z_{\nu}}+H_{H_{\nu}}\left(\mathbf{r}_{\nu}(t)\right)+H_{A_{\nu}}\left(\mathbf{r}_{\nu}(t)\right) .
$$

in which the stationary part

$$
\bar{H}_{Z_{\nu}}=\bar{g}_{\nu} \beta\left(\mathbf{S}_{\nu} \cdot \mathbf{B}\right)=\omega_{\nu} S_{\nu_{z}} \text { with } \omega_{\nu}=\bar{g}_{\nu} \beta B
$$

is the average Zeeman interaction of the electron spin $\mathbf{S}_{\nu}$ with the magnetic field $\mathbf{B}$. The fluctuating parts $H_{H_{\nu}}\left(\mathbf{r}_{\nu}(t)\right)$ and $H_{A_{\nu}}\left(\mathbf{r}_{\nu}(t)\right)$ represent HFI and AZI contributions, respectively.

Fluctuating HFI and AZI induce spin relaxation, which can be treated within the short correlation time approximation ${ }^{30}$ In this approximation the relaxation is described by the Bloch-Redfield equations for spin density matrices of polarons $\stackrel{30}{\underline{3}}$ The relaxation kinetics is determined by the relaxation supermatrices of the form

$$
\hat{W}_{Q_{\nu}}=w_{Q_{\nu}}^{p} \hat{P}_{\nu}^{p}+w_{Q_{\nu}}^{n} \hat{P}_{\nu}^{n}, \quad(Q=H, A),
$$

for both HFI $(H)$ and AZI $(A)$ mechanisms, where

$$
\begin{aligned}
\hat{P}_{\nu}^{p} & =\left(\left|\nu_{++}\right\rangle-\left|\nu_{--}\right\rangle\right)\left(\left\langle\nu_{++}\right|-\left\langle\nu_{--}\right|\right), \\
\hat{P}_{\nu}^{n} & =\left|\nu_{+-}\right\rangle\left\langle\nu_{+-}|+| \nu_{-+}\right\rangle\left\langle\nu_{-+}\right| .
\end{aligned}
$$

are the operators in the subspaces of diagonal $\left(\hat{P}_{\nu}^{p}\right)$ and non-diagonal $\left(\hat{P}_{\nu}^{n}\right)$ elements of the density matrix. The first and second terms in eq. (4.3) describe the population and phase relaxation, respectively, with rates 31

$$
w_{Q_{\nu}}^{p}=\bar{w}_{Q_{\nu}} \mathcal{J}_{Q}^{\nu}\left(\omega_{\nu}\right), \quad w_{Q_{\nu}}^{n}=\bar{w}_{Q_{\nu}}\left[p_{Q}+\mathcal{J}_{Q}^{\nu}\left(\omega_{\nu}\right)\right] .
$$

Here $p_{Q}$ is the numerical parameter, depending on the relaxation mechanism (specified by $Q=H, A$ ), $\bar{w}_{Q_{\nu}}$ is the characteristic rate (see below), and

$$
\mathcal{J}_{Q}^{\nu}(\omega)=\tau_{Q_{\nu}}^{-1} \int_{0}^{\infty} d t \Phi_{Q}^{\nu}(t) \cos (\omega t)
$$

with

$$
\tau_{Q_{\nu}}=\int_{0}^{\infty} d t \Phi_{Q}^{\nu}(t)
$$

is the normalized Fourier transformed correlation function satisfying the relation $\mathcal{J}_{\nu}(0)=1$.

The form of functions $\mathcal{J}_{Q}^{\nu}(\omega)$ depends on the mechanism of fluctuations. In the considered model of polaronhopping induced fluctuations these functions are essentially determined by hopping kinetics and specific features of the orientational distribution of molecules responsible for the HFI and AZI. In our work we will need only the most general properties of $\mathcal{J}_{Q}^{\nu}(\omega)$. They can be understood in a simple model of random Hamiltonians $H_{H_{\nu}}\left(\mathbf{r}_{\nu}\right)$ and $H_{A_{\nu}}\left(\mathbf{r}_{\nu}\right)$ uncorrelated at different sites (with zero mean values), in which $\Phi_{Q}^{\nu}(t) \sim \Phi_{\nu}(t)=$ $\left\langle\exp \left(-w_{E_{i} E_{j}}|t|\right)\right\rangle_{E_{i} E_{j}}$ and

$$
\mathcal{J}_{H}^{\nu}(\omega)=\left\langle w_{E_{i} E_{j}}\left(w_{E_{i} E_{j}}^{2}+\omega^{2}\right)^{-1}\right\rangle_{E_{i} E_{j}} /\left\langle w_{E_{i} E_{j}}^{-1}\right\rangle_{E_{i} E_{j}} .
$$

\section{A. Relaxation mechanisms}

\section{HFI and HFI induced relaxation}

The HFI $H_{H_{\nu}}$ is determined by the spin-spin interaction of the electron and paramagnetic nuclei $\nu_{j}$ (with spin $I_{\nu_{j}}$ ), localized in close surrounding of electronic spins $\mathbf{S}_{e}$ and $\mathbf{S}_{h}$ of $e$ - and $h$-polarons. In the realistic case of a large number of nuclei the interaction can quite accurately be approximated by that of spins $\mathbf{S}_{e}$ and $\mathbf{S}_{h}$ with (classical) random magnetic fields $\mathbf{B}_{e}$ and $\mathbf{B}_{h}$, respectively, whose distributions are isotropic and Gaussian with mean squares $\left\langle B_{\nu}^{2}\right\rangle \sim \sum_{j} I_{\nu_{j}}\left(1+I_{\nu_{j}}\right) a_{\nu_{j}}^{2},(\nu=e, h)$, determined by hyperfine coupling constants $a_{\nu_{j}}$.

Hopping of $e$ - and $h$-polarons results in sudden changing of nuclear magnetic field, which can be considered as a stochastic vector $\mathbf{B}_{\nu}(t),(\nu=e, h)$, with $\left\langle\mathbf{B}_{\nu}\right\rangle=0$ and the correlation function of projections $B_{\nu_{q}}(t),(q=$ $x, y, z):\left\langle B_{\nu_{q}}(t) B_{\nu_{q^{\prime}}}(0)\right\rangle=\frac{1}{3} \delta_{q q^{\prime}}\left\langle B_{\nu}^{2}\right\rangle \Phi_{H}^{\nu}(t)$.

The HFI mechanism predicts the relaxation superoperator $\hat{W}_{H_{\nu}}$ of the form (4.3) with the rates $w_{H_{\nu}}^{p}$ and $w_{H_{\nu}}^{n}$ given by eq. (4.6), in which

$$
p_{Q}=1 \quad \text { and } \quad \bar{w}_{H_{\nu}}=\frac{1}{3}\left(g_{\nu} \beta\right)^{2}\left\langle B_{\nu}^{2}\right\rangle \tau_{H_{\nu}},
$$

with the correlation time $\tau_{H_{\nu}}$ defined in eq. (4.8).

\section{2. $A Z I$ and $A Z I$ induced relaxation}

The AZI $H_{A}$ results from the deviation of $g_{\nu}$-factors of $e$ - and $h$-polaron spins from the free electron value $g_{0}=2 \cdot 30,31$ In general, $g$-factors are actually $\hat{g}_{\nu}$-tensors 31 whose eigenvectors are determined by the geometry of molecules, at which $e$ - and $h$-polarons are located. The $\hat{g}_{\nu}$-tensors are conveniently represented as sums of isotropic $\left(\bar{g}_{\nu}\right)$ and anisotropic $\left(\delta \hat{g}_{\nu}\right)$ parts:

$$
\hat{g}_{\nu}=\bar{g}_{\nu}+\hat{g}_{\nu}^{\prime}, \text { where } \bar{g}_{\nu}=\operatorname{Tr}\left(\hat{g}_{\nu}\right) .
$$

The representation (4.11) implies that $\operatorname{Tr}\left(\hat{g}_{\nu}^{\prime}\right)=0$. Usually, for systems under study eigenvalues of $\hat{g}_{\nu}$-tensors are close to $g_{0}=2: \delta \bar{g}_{\nu}=\bar{g}_{\nu}-g_{0} \sim\left\|\hat{g}_{\nu}^{\prime}\right\| \lesssim 10^{-2}$.

The AZI part of the interaction of the electron spin $\nu$ $(\nu=e, h)$ can be defined as

$$
H_{A_{\nu}}=\beta \mathbf{S}_{\nu} \hat{g}_{\nu} \mathbf{B}-\bar{H}_{Z_{\nu}}=\beta \mathbf{S}_{\nu} \hat{g}_{\nu}^{\prime} \mathbf{B} .
$$

In the AZI induced relaxation mechanism the rate superoperator $\hat{W}_{A_{\nu}}$ is written as (4.3) with rates $w_{A_{\nu}}^{p}$ and $w_{A_{\nu}}^{n}$ (4.6), in which 31

$$
p_{Q}=\frac{4}{3} \quad \text { and } \quad \bar{w}_{A_{\nu}}=\frac{1}{10}\left(\hat{g}_{\nu}^{\prime}: \hat{g}_{\nu}^{\prime}\right)(\beta B)^{2} \tau_{A_{\nu}},
$$

with $\tau_{A_{\nu}}$ given by eq. (4.8). 


\section{B. Hamiltonian and relaxation in polaron pairs}

The PP spin Hamiltonian is, in general, a complicated function of coordinates $\mathbf{r}_{h}$ and $\mathbf{r}_{e}$, determined by the electron spin dependent PP interactions: spin exchange and dipole-dipole interactions. Fortunately, the their effect on majority of MFEs is weak and can be neglected. ${ }^{19}$ In such a case the PP spin Hamiltonian can be represented as a sum of spin Hamiltonians of $e$ - and $h$-polarons:

$$
H=\bar{H}_{z_{e}}+\bar{H}_{Z_{h}}=\bar{g} \beta B S_{z}+\frac{1}{2} \Delta \bar{g} \beta B\left(S_{e_{z}}-S_{h_{z}}\right),
$$

where $S_{z}=S_{e_{z}}+S_{h_{z}}$,

$$
\bar{g}=\frac{1}{2}\left(\bar{g}_{e}+\bar{g}_{h}\right) \text { and } \Delta \bar{g}=\bar{g}_{e}-\bar{g}_{h} .
$$

Within similar assumptions, the $\mathrm{PP}$ spin relaxation can be described by the superoperator, which is also a sum of corresponding operators for separate polarons:

$$
\hat{W}=\hat{W}_{e}+\hat{W}_{h}=\sum_{\nu=e, h}\left(\hat{W}_{H_{\nu}}+\hat{W}_{A_{\nu}}\right) .
$$

The PP spin evolution is described in the four-state Hilbert space. In principle, one can use any basis in this space, for example the basis of states $\left|e_{\mu}\right\rangle\left|h_{\mu^{\prime}}\right\rangle,\left(\mu, \mu^{\prime}=\right.$ \pm ), of the pair of non-interacting polarons. However, in what follows it will be more convenient to use the basis of eigenstates of the $z$-projection, $S_{z}$, of the vector $\mathbf{S}$ : $|S\rangle,\left|T_{\mu}\right\rangle,(\mu=0, \pm)$, which correspond to the total; spin $S=0(S)$ and $S=1(T)$ :

$$
\begin{aligned}
& |S\rangle=\frac{1}{\sqrt{2}}\left(\left|e_{+}\right\rangle\left|h_{-}\right\rangle-\left|e_{-}\right\rangle\left|h_{+}\right\rangle\right), \\
& \left|T_{0}\right\rangle=\frac{1}{\sqrt{2}}\left(\left|e_{+}\right\rangle\left|h_{-}\right\rangle+\left|e_{-}\right\rangle\left|h_{+}\right\rangle\right), \\
& \left|T_{ \pm}\right\rangle=\left|e_{ \pm}\right\rangle\left|h_{ \pm}\right\rangle .
\end{aligned}
$$

In addition to the states in the Hilbert space it is worth introducing the states in the Liouville space

$$
|X Y\rangle=|X\rangle\langle Y|, \quad\left(X, Y=S, T_{\mu}\right) .
$$

\section{STOCHASTIC LIOUVILLE EQUATION}

The specific features of PP recombination processes (both geminate and bulk) can, in general, be expressed in terms the PP density matrix $\rho(\mathbf{r}, t)$. In the case of Markovian $e-h$ relative motion $\rho(\mathbf{r}, t)$ is known to satisfy the SLE, ${ }^{24,25}$ which within the diffusion approximation is written as

$$
\dot{\rho}=-[\hat{\Lambda}+\hat{K}(r)+\hat{L}] \rho, \text { where } \hat{\Lambda}=i \hat{H}+\hat{W},
$$

$\hat{H}=[H, \ldots]$ is the superoperator representation of the PP spin Hamiltonian $H$ [see eq. (4.14)] in the Liouville space, and $\hat{W}$ is the superoperator of $\mathrm{PP}$ spin relaxation, defined in eq. 4.16).

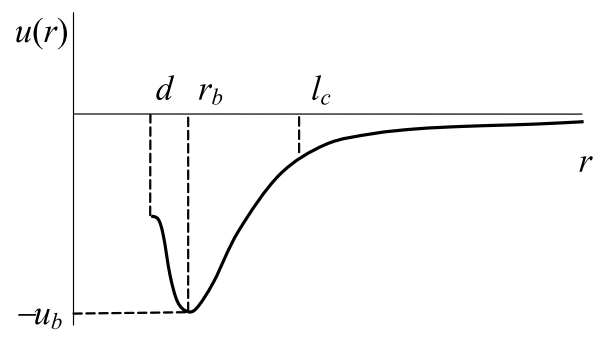

FIG. 1: Schematic picture of the PP interaction potential $u(r)=U(r) /\left(k_{B} T\right)$, in which $d$ is the distance of closest approach, $r_{b}$ is the coordinate of the well bottom, $u_{b}=-u\left(r_{b}\right)$ is the well depth, and $l_{c}$ is the Onsager radius.

The relative diffusive motion of $e$ - and $h$-polarons is described by the Smoluchowski operator $\hat{L}$, defined by the expression

$$
\hat{L} \rho=-D \nabla_{\mathbf{r}}\left(\nabla_{\mathbf{r}} \rho+\rho \nabla_{\mathbf{r}} u\right),
$$

in which $\mathbf{r}=\mathbf{r}_{h}-\mathbf{r}_{e}$ the relative $\mathrm{PPq}$ coordinate, $D=D_{h}+D_{e}$ is the relative diffusion coefficient [see eq. (2.1)], and $u(r)=U(r) /\left(k_{B} T\right)$ is the dimensionless $\mathrm{PP}$ interaction potential assumed to depend only on the distance $r=|\mathbf{r}|$.

In our analysis we will consider the spherically symmetric problem, thus reducing it to studying the evolution along the radial coordinate $r=|\mathbf{r}|=\left|\mathbf{r}_{h}-\mathbf{r}_{e}\right|$ only.

The potential $u(r)$ is assumed to be of the shape of potential well (see Fig. 1). This potential is characterized by the barrier, of height $u_{r}=u(d)-u\left(r_{b}\right)>1$, at the distance $d$ of closest approach of polarons (which models the suggested smallness of the reaction rate), the coordinate $r_{b}$ of the bottom $\left(r_{b}>d\right)$, the well depth $u_{b}=-u\left(r_{b}\right)>1$, as well as the Onsager radius

$$
l_{c}=\int_{r_{b}}^{\infty} d r r^{-2} e^{u(r)} .
$$

The term $\hat{K}(r) \rho$ describes spin selective recombination assumed to occur only in $S$-state of the PP:19,24

$$
\hat{K}(r)|\rho\rangle=\frac{1}{2} \kappa(r)\left(P_{S} \rho+\rho P_{S}\right) \equiv \kappa(r) \hat{\mathcal{P}}_{S}|\rho\rangle,
$$

where $\kappa(r)$ is the distance dependent recombination rate. In eq. (5.4) $P_{S}=|S\rangle\langle S|$ is the operator of projection on the $S$-state and

$$
\hat{\mathcal{P}}_{S}=\hat{P}_{S S}+\frac{1}{2} \sum_{\mu=0, \pm}\left(\hat{P}_{S T_{\mu}}+\hat{P}_{T_{\mu} S}\right)
$$

is the superoperator, controlling the spin dependence of reactivity (and accompanying dephasing), in which

$$
\hat{P}_{X Y}=|X Y\rangle\langle X Y|, \quad\left(X, Y=S, T_{\mu}\right),
$$

are superoperators of projection onto the states (4.20) in the Liouville space. The dependence $\kappa(r)$ is typically 
short range and does not affect MFEs very much, so that, for simplicity, one can apply the contact reactivity model

$$
\kappa(r)=\kappa_{S} \delta(r-d) \text {, i.e. } \hat{K}(r)=\hat{K}_{S} \delta(r-d)
$$

with $\hat{K}_{S}=\kappa_{S} \hat{\mathcal{P}}_{S}$.

The polarons are assumed to reflect at the distance of closest approach $d$. The process is described by the reflective inner boundary condition $\left(\nabla_{r} \rho+\rho \nabla_{r} u\right)_{r=d}=0$. In the model of contact reactivity (5.7), however, one can formally reduce the effect of reactivity to the modification of the inner boundary condition:

$$
\left.\left[\nabla_{r} \rho+\rho \nabla_{r} u-\left(\hat{K}_{S} / D\right) \rho\right)\right]_{r=d}=0 .
$$

As for the outer boundary condition it is different for geminate and bulk processes (see below).

\section{GEMINATE PP RECOMBINATION}

The kinetics of geminate $\mathrm{PP}$ recombination will be analyzed assuming that PPs are created with isotropic distribution, localized at a distance $r=r_{i}$, and in the spin state, determined by the density matrix $\rho_{i}(r)$. This means that the SLE (5.1) should be solved with the initial condition

$$
\rho_{i}(r)=\rho(\mathbf{r}, t=0)=\left(4 \pi r_{i}^{2}\right)^{-1} \rho_{g} \delta\left(r-r_{i}\right) .
$$

The outer boundary condition at $r \rightarrow \infty$, is written as $\rho(r \rightarrow \infty, t) \rightarrow 0$.

Spherically symmetry of PP interactions, as well as spherical symmetry of initial and boundary conditions ensures that the spin density matrix is also spherically symmetric: $\rho(\mathbf{r}, t) \equiv \rho(r, t)$. In this case the PP recombination yield $Y_{r}$, can be expressed by formula

$$
\begin{aligned}
Y_{r} & =\int_{0}^{\infty} d t \int d^{3} r \operatorname{Tr}[\hat{K}(r) \rho(r, t)] \\
& =4 \pi \kappa_{s} d^{2}\left\langle S\left|\widetilde{\rho}_{0}(d)\right| S\right\rangle \equiv 4 \pi \kappa_{s} d^{2}\left\langle S S \mid \widetilde{\rho}_{0}(d)\right\rangle,
\end{aligned}
$$

where the matrix $\widetilde{\rho}_{0}(r)$ is the Laplace transform $\widetilde{\rho}_{\epsilon}(r)=$ $\int_{0}^{\infty} d t e^{-\epsilon t} \rho(r, t)$, evaluated at $\epsilon=0: \widetilde{\rho}_{0}(r)=\widetilde{\rho}_{\epsilon=0}(r)$.

The matrix $\widetilde{\rho}_{0}(r)$ satisfies the steady state variant of the SLE 15.1

$$
\left(\hat{\Lambda}+\hat{L}_{r}\right) \widetilde{\rho}_{0}=\rho_{i}(r) \text {, where } \hat{\Lambda}=i \hat{H}+\hat{W},
$$

and $L_{r}$ is the radial part of the operator $\hat{L}$ defined by

$$
L_{r} \rho=D r^{-2} \nabla_{r}\left[r^{2}\left(\nabla_{r} \rho+\rho \nabla_{r} u\right)\right] .
$$

Noteworthy is that in the SLE (6.3) we have omitted the reactivity operator $\hat{K}(r)$ (5.7), expressing its effect by the properly chosen inner boundary condition (5.8).

For the initial condition (6.1) the solution of this equation is easily expressed in terms of the Green's function of the equation (6.3)

$$
\hat{G}_{0}=\left(\hat{\Lambda}+\hat{L}_{r}\right)^{-1}:
$$

$$
Y_{r}=\kappa_{s}\left(d / r_{i}\right)^{2}\left\langle S S\left|\hat{G}_{0}\left(d, r_{i}\right)\right| \rho_{g}\right\rangle
$$

In general, $\hat{G}_{0}\left(r, r_{i}\right)$ can hardly be obtained analytically. However, fairly simple analytical expressions for $\hat{G}_{0}\left(r, r_{i}\right)$ (and thus for $Y_{r}$ ) can be found in some important particular cases.

\section{A. Freely diffusing polarons.}

\section{General formulas}

In the absence of PP interaction potential $[u(r)=0]$ the solution of eq. 6.5 can be obtained in a matrix form. 25,33 For simplicity we will assume that PPs are created at a distance of closest approach, i.e. $r_{i}=d$.

The analytical expression can conveniently be obtained with the use of the representation

$$
\hat{G}_{0}=D^{-1}\left(r_{i} / r\right) \hat{g}_{0}
$$

In this relation

$$
\hat{g}_{0}=\left(\hat{k}_{0}^{2}-\nabla_{r}^{2}\right)^{-1}, \text { with } \hat{k}_{0}=(\hat{\Lambda} / D)^{1 / 2},
$$

is the one dimensional Green's function, which satisfies the inner boundary condition similar to (5.8):

$$
\left.\left(\nabla_{r}-\hat{q}_{s}\right) \hat{g}_{0}\right|_{r=d}=0 \text { with } \hat{q}_{s}=d^{-1}+\left(\hat{K}_{S} / D\right) .
$$

The function $\hat{g}_{0}$ is given by formula ${ }^{25,33}$

$$
\hat{g}_{0}\left(r, r^{\prime}\right)=\left[e^{-\hat{k}_{0}(r-d)} \hat{\lambda}+e^{-\hat{k}_{0}\left|r-r^{\prime}\right|}\right]\left(2 \hat{k}_{0}\right)^{-1},
$$

where

$$
\hat{\lambda}=(\hat{\theta}+1)^{-1}(\hat{\theta}-1) e^{-\hat{k}_{0}\left(r^{\prime}-d\right)} \text { and } \hat{\theta}=\hat{q}_{s}^{-1} \hat{k}_{0} .
$$

Substitution of this formula into eqs. (6.8) and (6.2) results in the expression for the recombination yield:

$$
Y=Y_{f}=\operatorname{Tr}\left[P_{S}\left(\hat{\mathbf{P}}_{f} \rho_{g}\right)\right] \equiv\left\langle S S\left|\hat{\mathbf{P}}_{f}\right| \rho_{g}\right\rangle,
$$

in which

$$
\hat{\mathbf{P}}_{f}=\hat{\mathcal{P}}_{r_{f}}\left(1+d \hat{k}_{0} \hat{\mathcal{P}}_{e_{f}}\right)^{-1},
$$

is the spin dependent supermatrix of reaction/relaxation probabilities. In eq. 6.13

$$
\hat{\mathcal{P}}_{r_{f}}=\hat{l} / d \text { and } \hat{\mathcal{P}}_{e_{f}}=1-\hat{l} / d,
$$

are the supermatrices of probabilities of reaction and escaping (respectively) of PPs, created at $r_{i}=d$, in the absence of PP spin evolution. The probabilities are essentially determined by the supermatrix $\hat{l}$ of reaction/relaxation radii, corresponding to the rate supermatrix $\hat{K}(r)$ [given by eq. (5.7)]:

$$
\hat{l}=d-\hat{q}_{s}^{-1}=d \hat{\gamma}_{f} /\left(1+\hat{\gamma}_{f}\right) \text { with } \hat{\gamma}_{f}=d \hat{K}_{S} / D \text {. }
$$

Formulas (6.12)-6.15) for the yield of recombination of freely diffusing polarons are seen to reduce the problem of evaluation of MFEs to simple matrix operations. 


\section{Fast P-diffusion limit}

The limit of fast relative diffusion (or slow spin evolution), when $d\left\|\hat{k}_{0}\right\| \ll 1, \stackrel{25,33}{ }$ is of special interest for our further analysis. In this limit $\hat{\mathbf{P}}_{f}$ can be found in the approximation linear in $d \hat{k}_{0}: \hat{\mathbf{P}}_{f} \approx \hat{\mathcal{P}}_{r_{f}}\left(1-d \hat{k}_{0} \hat{\mathcal{P}}_{e_{f}}\right)$, i.e.

$$
Y_{f} \approx\left(l_{S S} / d\right) p_{S}-l_{S S}\left\langle S S\left|\hat{k}_{0} \hat{\mathcal{P}}_{e_{f}}\right| \rho_{g}\right\rangle,
$$

where $l_{S S}$ is the reaction radius in $S$-state and $p_{S}=$ $\left\langle S S \mid \rho_{g}\right\rangle$ is the initial population of this state.

For the model (4.14), (4.16) the yield $Y_{f}$ can be obtained for any initial state $\left|\rho_{g}\right\rangle$ and, in particular, for

$$
\left|\rho_{g}\right\rangle=p_{S}\left|\rho_{S}\right\rangle+p_{T}\left|\rho_{T}\right\rangle
$$

in which $p_{S}$ and $p_{T}$ are the probabilities of population of $S$ and $T$ states, respectively $\left(p_{S}+p_{T}=1\right)$,

$$
\left|\rho_{S}\right\rangle=|S S\rangle \text { and }\left|\rho_{T}\right\rangle=\frac{1}{3} \sum_{\mu}\left|T_{\mu} T_{\mu}\right\rangle
$$

Calculation of $Y_{f}$ reduces to evaluating the superma$\operatorname{trix} \hat{k}_{0}=(2 \sqrt{\pi D})^{-1} \int_{0}^{\infty} d t t^{-3 / 2}\left(1-e^{-i \hat{\Lambda} t}\right)$. Substituting thus obtained $\hat{k}_{0}$ into eq. (6.16), one gets

$$
Y_{f} \approx \mathcal{P}_{r_{f}}^{S}\left[p_{S}+\frac{1}{12}\left(d k_{w}\right)\left(p_{T} \mathcal{P}_{e_{f}}^{T}-3 p_{S} \mathcal{P}_{e_{f}}^{S}\right)\right],
$$

where $\mathcal{P}_{e_{f}}^{T}=1-l_{T T} / d=1, \mathcal{P}_{r_{f}}^{S}=1-\mathcal{P}_{e_{f}}^{S}=l_{S S} / d$, and

$$
k_{w}=\left[\sqrt{w_{p}}+2 \operatorname{Re}\left(\sqrt{w_{n}+i \Delta \omega}\right)\right] / \sqrt{D} .
$$

In this formula

$$
\Delta \omega=\left(\bar{g}_{e}-\bar{g}_{h}\right) \beta B=\delta \bar{g} \beta B .
$$

is the difference of Zeeman polaron frequencies and

$$
w_{p}=2\left(w_{H}^{p}+w_{A}^{p}\right) \text { and } w_{n}=w_{H}^{n}+w_{A}^{n},
$$

are the total rates of population $\left(w_{p}\right)$ and phase $\left(w_{n}\right)$ relaxation in the $\mathrm{PP}$, which are the sums of contributions of the HFI and AZI induced relaxation rates. These contributions are, in turn, the sums of corresponding relaxation rates in $e$ - and $h$-polarons:

$$
w_{H}^{q}=w_{H_{e}}^{q}+w_{H_{h}}^{q} ; w_{S}^{q}=w_{A_{e}}^{q}+w_{A_{h}}^{q},(q=p, n) .
$$

The total rates $w_{p}$ and $w_{n}$ are represented as sums of HFI and AZI induced relaxation rates to clearly reveal two contributions with different $B$-dependence: decreasing $\left[w_{H_{\nu}}^{q}(B)\right]$ and increasing $\left[w_{A_{\nu}}^{q}(B)\right]$ as $B$ increases.

\section{B. PP recombination in the presence of interaction}

The PP interaction potential $u(r)$ can essentially affect the MFEs on PP recombination. For pure repulsive interaction $[u(r)>0]$ no significant effect is expected except some change of reaction and relaxation radii, i.e. the elements of the supermatrix $\hat{l}$ introduced in eq. 6.15) for freely diffusing polarons. Much stronger effect is predicted in the case of attractive interaction, especially for potentials of the shape of potential well (Fig. 1) $\stackrel{\underline{34}}{=} \mathrm{In}$ what follows we will discuss the MFEs in the special case of deep potential well with $u_{b} \gg 1$.

The analysis can be made with the use of the recently proposed method of rigorous analytical analysis of the kinetics of diffusion in the presence of the well. 26 One of important results of this analysis consists in the fact that in the limit of deep well (corresponding criterion is given below) the exact SLE (5.1) is equivalent to the model of two kinetically coupled states: the state within the well (the diffusive cage state), at $d<r<l_{c}$, and the state of free diffusion outside the well, i.e. at $r>l_{c} \stackrel{26,27}{\underline{20}}$ The spin/space evolution in these two states are described by density matrices

$$
n(t)=4 \pi \int_{d}^{l_{c}} d r r^{2} \rho(r, t) \text { and } \sigma(r, t)=r \rho(r, t),
$$

respectively, satisfying equations $\underline{26,27}$

$$
\begin{aligned}
& \dot{n}=\left[S_{l}^{-1} K_{+} \sigma\left(l_{c}, t\right)-\left(K_{-}+\hat{W}_{r}\right) n\right], \\
& \dot{\sigma}=\left[D \nabla_{r}^{2} \sigma+\left(S_{l} K_{-} n-K_{+} \sigma\right) \delta\left(r-l_{c}\right)\right],
\end{aligned}
$$

in which $S_{l}=\left(4 \pi l_{c}\right)^{-1}$. The terms proportional to $K_{ \pm}$represent the kinetic coupling (transitions) between the two states, with transition rates $K_{ \pm}$satisfying the relations:27 $K_{ \pm} \rightarrow \infty$ and $K_{+} / K_{-}=K_{e}=Z_{w}$, where

$$
Z_{w}=\int_{d<r<l_{c}} d r r^{2} e^{-u(r)}
$$

is the partition function for the well.

Equations (6.25) and (6.25) will be solved assuming that the PP is initially created within the well, i.e.

$$
n(0)=\rho_{g} \quad \text { and } \quad \sigma(r, 0)=0 .
$$

As to the boundary conditions for $c(r, t)$, they are given by $l_{c} \nabla_{r} \sigma(r, t)-\left.\sigma(r, t)\right|_{r=l_{c}}=0$ and $\sigma(r \rightarrow \infty)=0$.

The term $\hat{W}_{r} \widetilde{n}$ in eq. (6.25) describes the first order reaction in the well with the supermatrix of rates

$$
\hat{W}_{r}=\left(\frac{D}{\kappa_{r} Z_{w}}\right) \frac{\hat{\gamma}_{c}}{1+\hat{\gamma}_{c}} \text { with } \kappa_{r}=\int_{d}^{r_{b}} \frac{d r}{r^{2}} e^{u(r)}
$$

and $\hat{\gamma}_{c}=\left(d \hat{K}_{S} / D\right)\left(d \kappa_{r} e^{-u(d)}\right)$.

Solution of eqs. (6.25) and (6.26) by the Laplace transformation in time leads to formula for the recombination yield $^{34}$ similar to eq. (6.12):

$$
Y_{c}=\operatorname{Tr}\left[P_{S}\left(\hat{\mathbf{P}}_{c} \rho_{g}\right)\right] \equiv\left\langle S S\left|\hat{\mathbf{P}}_{c}\right| \rho_{g}\right\rangle,
$$

but with the supermatrix $\hat{\mathbf{P}}_{f}$ replaced by

$$
\begin{aligned}
\hat{\mathbf{P}}_{c} & =\hat{W}_{r}\left[\hat{W}_{c}+\hat{\Lambda}+W_{e}\left(l_{c} \hat{k}_{0}\right)\right]^{-1}, \\
& =\hat{\mathcal{P}}_{r_{c}}\left[1+\left(\hat{\Lambda} / W_{e}+l_{c} \hat{k}_{0}\right) \hat{\mathcal{P}}_{e_{c}}\right]^{-1},
\end{aligned}
$$


in which $\hat{\Lambda}=i \hat{H}+\hat{W}, \hat{k}_{0}=(\hat{\Lambda} / D)^{1 / 2}$, and

$$
\hat{W}_{c}=\hat{W}_{r}+W_{e}, \quad \text { with } W_{e}=D l_{c} / Z_{w},
$$

is the supermatrix of total cage decay rates, represented as a sum of the rate $\hat{W}_{r}$ of reaction in the well (cage) and the rate $W_{e}$ of escaping from the well $\stackrel{26,27}{2}$ These rates determine the probabilities of reaction in the well $\left(\hat{\mathcal{P}}_{r_{c}}\right)$ and escape from the well $\left(\hat{\mathcal{P}}_{e_{c}}\right)$ :

$$
\hat{\mathcal{P}}_{r_{c}}=\hat{W}_{r} / \hat{W}_{c} \text { and } \hat{\mathcal{P}}_{e_{c}}=W_{e} / \hat{W}_{c} .
$$

The detailed analysis shows 26 that expressions (6.31) and (6.32) are valid in the limit of not very fast PP spin evolution, or not very large size of the well $\delta_{c}=l_{c}-d$, when $\delta_{c}\left\|\hat{k}_{0}\right\| \ll 1$ (this inequality does not, in principle, mean that $\left.l_{c}\left\|\hat{k}_{0}\right\| \ll 1\right)$.

According to obtained formulas, the effect of the well manifests itself in the formation of the diffusive cage, whose evolution is described as the first order reaction (with the rate $\hat{W}_{r}$ ) and escaping (with the rate $W_{e}$ ). This simple first order kinetics is, however, perturbed by the contribution of particles escaped but recaptured back into the well, resulting in the term $\sim\left(l_{c} \hat{k}_{0}\right)$ in eqs. (6.31) and (6.32), which gives negligibly small contribution to MFEs for deep wells (when $W_{e}$ is so small that $\left.\|\hat{\Lambda}\| / W_{e}>l_{c}\left\|\hat{k}_{0}\right\|\right)$, but strongly affects MFEs in the opposite limit of large escaping rate $W_{e}$. Moreover in the limit of fast cage decay (or slow PP spin evolution), $\|\hat{\Lambda}\| / W_{e} \ll l_{c}\left\|\hat{k}_{0}\right\|$, the obtained formula reduces to that (6.13) for free diffusion. 26

\section{BULK PP RECOMBINATION}

The MFEs in bulk recombination are also described by the SLE (5.1) but with the boundary condition at $r \rightarrow \infty$

$$
\rho(r \rightarrow \infty, t)=\rho_{E}=\frac{1}{4} \sum_{\mu=S, T_{0, \pm}}|\mu\rangle\langle\mu|,
$$

corresponding to the homogeneous spatial distribution of and the equilibrium spin states of polarons [represented by the unity matrix $\left.E_{H}=\sum_{\mu=S, T_{0, \pm}}|\mu\rangle\langle\mu|\right]$.

In the case of bulk recombination the observable under study is $B$-dependent recombination rate $K(t)$. For simplicity we will discuss the static value $K=K(t \rightarrow \infty)$ which can be represented in the form very similar to that of the expression (6.2) for the recombination yield:

$$
\begin{aligned}
K & =\int d^{3} r \operatorname{Tr}[\hat{K}(r) \rho(r, t \rightarrow \infty)] \\
& =4 \pi \kappa_{s} d^{2}\left\langle S\left|\widetilde{\rho}_{0}(d)\right| S\right\rangle \equiv 4 \pi \kappa_{s} d^{2}\left\langle S S \mid \widetilde{\rho}_{0}(d)\right\rangle,
\end{aligned}
$$

This formula is written, taking into account that the equation for the static solution $\rho_{s t}(r)$ coincides with the Laplace transform $\widetilde{\rho}_{\epsilon=0}(r) \equiv \widetilde{\rho}_{0}(r)$ [however, with the outer boundary condition (7.1)]: $\rho_{s t}(r)=\widetilde{\rho}_{0}(r)$.

\section{A. Freely diffusing polarons.}

The stationary solution $\widetilde{\rho}_{0}(r)$ of the SLE (5.1) satisfying eq. (6.3) with $\rho_{i}=0$ can be found by solving more simple equation for $\widetilde{\sigma}_{0}(r)=r^{-1} \widetilde{\rho}_{0}(r)$

$$
\left(\hat{k}_{0}^{2}-\nabla_{r}^{2}\right) \widetilde{\sigma}_{0}=0 \text { with } \hat{k}_{0}=(\hat{\Lambda} / D)^{1 / 2} .
$$

The function $\widetilde{\sigma}_{0}(r)$ satisfies the inner boundary condition $\left.\left(\nabla_{r}-\hat{q}_{s}\right) \widetilde{\sigma}_{0}\right|_{r=d}=0$, similar to (6.9), and the outer one, which, according to eq. (7.1), is written as

$$
\tilde{\sigma}_{0}(r \rightarrow \infty)=r \rho_{E} .
$$

By direct substitution into eq. (7.3) one can show that the solution of this equation is given by

$$
\widetilde{\sigma}_{0}(r)=\left[-e^{-\hat{k}(r-d)}(1+\hat{\theta})^{-1} \hat{l}+r\right] \rho_{E} .
$$

where $\hat{\theta}$ is defined in eq. (6.11). With the use of this solution the recombination rate is represented as

$$
K=K_{f}=K_{f}^{0} Y_{f_{E}}, \text { where } Y_{f_{E}}=\left\langle S S\left|\hat{\mathbf{P}}_{f}\right| \rho_{E}\right\rangle,
$$

In this formula $Y_{f_{E}}$ is the geminate recombination yield for the equilibrium initial $\rho_{g}=\rho_{E}, \hat{\mathbf{P}}_{f}$ is the superoperator of reaction/relaxation probabilities defined in eq. (6.13), and $K_{f}^{0}=4 \pi D d$ is the rate of PP contacts.

Note that the dimensionless rate $K_{f} / K_{f}^{0}$ can be related to the yield $Y_{f_{T}}$ of the geminate $\mathrm{PP}$ recombination for (triplet) initial density matrix $\rho_{g}=\rho_{T}$ :

$$
K_{f} / K_{f}^{0}=Y_{f_{E}}=\frac{1}{4} \mathcal{P}_{r_{f}}^{s}\left(1+3 Y_{f_{T}}\right),
$$

where $\mathcal{P}_{r_{f}}^{S}=l_{S S} / d$ is the PP recombination probability in $S$ state. Equation (7.7) is obtained using the relation $\hat{\mathbf{P}}_{f}=\hat{\mathcal{P}}_{r_{f}}\left(1+d \hat{k}_{0} \hat{\mathcal{P}}_{e_{f}}\right)^{-1}=\hat{\mathcal{P}}_{r_{f}}-\hat{\mathbf{P}}_{f}\left(d \hat{k}_{0} \hat{\mathcal{P}}_{e_{f}}\right)$.

\section{B. Interacting polarons}

In the considered case of attractive interaction the expression for the PP recombination rate $K_{c}$ can be obtained within the two state model (Sec. VI.B). For bulk reactions the spin density matrix, represented in terms of spin density matrices of the state within the well $n_{0}$ and the free diffusion state $\sigma_{0}(r)$, satisfy the steady state variant of eqs. (6.25) and (6.26), i.e. equations with $\dot{n}_{0}=\dot{\sigma}_{0}=0$, but with the outer boundary condition: $\sigma_{0}(r \rightarrow \infty)=r \rho_{E}$.

Solution of these steady state equations leads to the following expression for the $\mathrm{PP}$ recombination rate

$$
K=K_{c}=K_{c}^{0} Y_{c_{E}} \text { with } Y_{c_{E}}=\left\langle S S\left|\hat{\mathbf{P}}_{c}\right| \rho_{E}\right\rangle,
$$

in which $Y_{c_{E}}$ is $\mathrm{PP}$ recombination yield for the equilibrium (spin) initial state, $\hat{\mathbf{P}}_{c}$ is given by eq. (6.31), and $K_{c}^{0}=4 \pi D l_{c}$ is the rate of capture into the well (cage). 
The relation between the dimensionless rate $K_{c} / K_{c}^{0}$ and the recombination yield $Y_{c_{T}}$ [for the triplet $(T)$ initial condition], similar to eq. (7.7), can also be derived in the case of attractive interaction:

$$
K_{c} / K_{c}^{0}=Y_{c_{E}}=\frac{1}{4} \mathcal{P}_{r_{c}}^{S}\left(1+3 Y_{c_{T}}\right),
$$

where $\mathcal{P}_{r_{c}}^{S}=\left\langle S S\left|\left(\hat{W}_{r} / \hat{W}_{c}\right)\right| S S\right\rangle$ is the probability of incage reaction in $S$ state. The derivation is based on equation $\hat{\mathbf{P}}_{c}=\hat{\mathcal{P}}_{r_{c}}-\hat{\mathbf{P}}_{c}\left[\hat{\Lambda} / W_{e}+\left(l_{c} \hat{k}_{0}\right)\right] \hat{\mathcal{P}}_{e_{c}}$, with $\hat{\mathcal{P}}_{r_{c}}$ and $\hat{\mathcal{P}}_{e_{c}}$ defined in eq. (6.34).

\section{RESULTS AND DISCUSSION}

\section{A. General remarks \\ 1. Validity of approaches}

Before the analysis of the MFEs it is worth adding some comments on validity and accuracy of the proposed mechanisms of spin relaxation. In the applied model the relaxation, assumed to be induced by fluctuating HFI and AZI, is described by simple Bloch-type equations, which are valid at times longer than the correlation times $\tau_{h}$ and $\tau_{a}$ of HFI and AZI fluctuations. This means that the relaxation mechanisms are applicable only if the time of MFE formation $\tau_{S} \gg \tau_{h}, \tau_{a}$.

In the free diffusion model the MFE-formation time $\tau_{S}$ is completely determined by the time of spin evolution in polarons: $\tau_{S} \sim\|\hat{\Lambda}\|^{-1} \sim w_{n}^{-1}, w_{p}^{-1},(\Delta \omega)^{-1} \underline{25}$ Taking into account that the relaxation rates $\left(w_{H}\right.$ and $\left.w_{A}\right)$ and coherent evolution frequencies $(\sim \Delta \omega)$ ] are typically of order of (or less than) $10^{8} \mathrm{~s}^{-1} \stackrel{19}{=}$ one obtains: $\tau_{S} \gtrsim 10^{-8} \mathrm{~s}$.

Similar estimation for $\tau_{S}$ is valid in the diffusive cage model. The additional time parameter, which could change it, is the characteristic inverse rate of the cage decay $\tau_{c}=\left\|\hat{W}_{c}\right\|^{-1}$. However, this time is also fairly long: $\tau_{c} \gtrsim w_{0}^{-1} e^{u_{a}}$, where $w_{0}$ is the characteristic hopping rate and $u_{a}$ is the activation energy for escaping and/or reaction processes. The assumption of deep well $\left(u_{a} \gg 1\right)$ leads to the estimation $\tau_{c} \gg w_{0}^{-1}$.

As for correlation times they are expected to be of order of inverse hopping rates: $\tau_{h} \sim \tau_{a} \sim w_{0}^{-1}$, since HFI and AZI fluctuations result from stochastic polaron hopping. Typically the rate $w_{0} \sim 10^{9}-10^{11} \mathrm{~s}^{-1}$, which corresponds to room-temperature mobilities $\mu_{e, h} \sim 10^{-8}-$ $10^{-5} \mathrm{~cm}^{2} /(\mathrm{V} \mathrm{s}), \frac{17}{,}$ therefore we get $\tau_{h, a} \sim 10^{-9}-10^{-11} \mathrm{~s}$.

Comparison of these estimations shows that the abovementioned validity criterion, $\tau_{S}, \tau_{c} \gg \tau_{h}, \tau_{a}$, is fulfilled for a large number of semiconducting systems considered.

Concluding the discussion note that the validity of the diffusion approximation implies negligibly small contribution (to the MFEs) of small times $t \sim \tau_{0}=w_{0}^{-1}$, at which the diffusive motion is not yet formed. In the considered $f$-and $c$-models, according to the above relations, their MFE contributions $\delta Y_{\mu}=Y_{\mu}(B)-Y_{\mu}(0),(\mu=$ $f, c)$, can be written as $\delta Y_{f} \sim\left(w_{0} \tau_{S}\right)^{-1 / 2}$ and $\delta Y_{c} \sim$ $\tau_{c} / \tau_{S} \sim e^{u_{a}} /\left(w_{0} \tau_{S}\right)$. As to the contribution $\delta Y_{s m}$ of small times $t \sim \tau_{0}$, it is, evidently, represented by $\delta Y_{s t} \sim\left(w_{0} \tau_{S}\right)^{-1}$, i.e. in the considered limit $w_{0} \tau_{S} \ll 1$ the contribution $\delta Y_{s t}$ is really small: $\delta Y_{s t} \ll \delta Y_{f}, \delta Y_{c}$ and the diffusion approximation is applicable.

\section{Parameters and observables}

In the proposed models $B$-dependence of the recombination yield $Y(B)$ is determined by a very large number of parameters of the model. To reduce this number in our illustrative discussion of most important properties of $Y(B)$, we consider the particular (but representative) variant of the model, in which population and phase relaxation rates ( $w^{p}$ and $w^{n}$, respectively) are the same in $e$ - and $h$-polarons:

$$
w_{Q_{e}}^{p}=w_{Q_{h}}^{p}=\frac{1}{2} w_{Q}^{p} ; \quad w_{Q_{e}}^{n}=w_{Q_{h}}^{n}=\frac{1}{2} w_{Q}^{n},
$$

with $Q=H, A$; and

$$
w_{Q}^{p}=\bar{w}_{Q} \mathcal{J}_{Q}(\omega) ; \quad w_{Q}^{n}=p_{Q} \bar{w}_{Q}+w_{Q}^{p} .
$$

In formulas (8.1) and (8.2) the parameter $Q$ denotes the contributions of HFI $(Q=H)$ and AZI $(Q=A)$ mechanisms, and the parameters $p_{Q}$ and $\bar{w}_{Q}$ are defined in eqs. (4.10) and (4.13). In these formulas we neglect the difference of Zeeman frequencies in functions $\mathcal{J}_{Q}\left(\omega_{\nu}\right)$, i.e. took $\omega_{e} \approx \omega_{h}=\omega=\bar{g} \beta B$.

The MFEs on geminate and bulk PP recombination are found to be closely related [see eqs. (7.6)-(7.9)], so that it is sufficient to analyze, for example, the MFE on bulk process, i.e. $B$-dependent (dimensionless) rate $K_{\mu}(B) / K_{\mu}^{0}=Y_{\mu_{E}}(B)$. In what follows we will discuss the function

$$
y_{\mu}(B)=\left[Y_{\mu_{E}}(B)-Y_{\mu_{E}}(0)\right] / Y_{\mu_{E}}(0), \quad(\mu=f, c) .
$$

In our work we have obtained fairly simple matrix expressions for the MFE $y_{\mu}(B)$ in two models of relative polaron motion. For qualitative understanding of the properties of these functions, however, it is of certain interest to get simple approximate analytical expressions. Below we will derived them in some limiting models.

\section{B. Simple limiting models}

\section{Fast freely diffusing polarons.}

In the case of freely diffusing polarons of special interest is the limit of fast polaron diffusion or slow polaron spin evolution, in which $\left\|\hat{k}_{0}\right\| d \ll 1$.

General consideration of the problem predicts for the recombination yield the expression (6.19). In what follows we will mainly discuss the shape of the MFE $Y_{f_{E}}$, which can be written as

$$
Y_{f_{E}} \approx \frac{1}{4} \mathcal{P}_{r_{f}}^{S}+\frac{1}{16}\left(\mathcal{P}_{r_{f}}^{S}\right)^{2}\left(d k_{w}\right)
$$


where $k_{w}=\left[\sqrt{w_{p}}+2 \operatorname{Re}\left(\sqrt{w_{n}+i \Delta \omega}\right)\right] / \sqrt{D}$ [see eq. [6.20] $], \mathcal{P}_{r_{f}}^{S}=l_{S S} / d$ is recombination probability in $S$ state, $w_{p}$ and $w_{n}$ are population and phase relaxation rates defined in eqs. (6.22) and (6.23), respectively, and $\Delta \omega=\left(\bar{g}_{e}-\bar{g}_{h}\right) \beta B=(\Delta \bar{g}) \beta B$ is the difference of Zeeman frequencies [eq. [6.21)]. According to formulas (6.22) and (6.23) the rates $w_{p}$ and $w_{n}$ are the sums of the corresponding rates for $e$ and $h$ polarons, which are, in turn, the sums of contributions of HFI and AZI mechanisms: $w_{p}=2\left(w_{H}^{p}+w_{A}^{p}\right)$ and $w_{n}=w_{H}^{n}+w_{A}^{n}$ so that

$$
\begin{aligned}
w_{p}(B) & =2\left[\bar{w}_{H} \mathcal{J}_{H}(B)+\bar{w}_{A}(B) \mathcal{J}_{A}(B)\right], \\
w_{n}(B) & =\bar{w}_{H}+\frac{4}{3} \bar{w}_{A}(B)+\frac{1}{2} w_{p}(B) .
\end{aligned}
$$

In these equations we have introduced the function $\bar{w}_{A}(B)$ to emphasize that (unlike $\bar{w}_{H}$ ) $\bar{w}_{A}$ depends on $B$ [see eq. [4.14)]: $\bar{w}_{A}(B) \sim B^{2}$.

Noteworthy is that, although formula (8.4) is derived in the fast diffusion limit $d k_{w} \ll 1$, it appears to be quite accurate (within $15-20 \%$ ) even at $d k_{w} \sim 1$.

\section{The limit of weak reactivity in the well.}

Majority of specific features of MFEs in the presence of the well (diffusive cage) can be analyzed with the limit of weak reactivity, when $\zeta_{c}=\left\|\hat{W}_{r}\right\| / W_{e} \ll 1$. In this limit the expression for the yield $Y_{c_{E}}$ can be derived by expansion of the general formulas (6.30), (6.31), and (7.9) in small $\zeta_{c}$. In the lowest order we get

$$
\begin{aligned}
Y_{c_{E}} \approx & \frac{1}{4} \mathcal{P}_{r_{c}}^{S} \\
& +\frac{1}{16}\left(\mathcal{P}_{r_{c}}^{S}\right)^{2}\left\{N\left(w_{p}\right)+2 \operatorname{Re}\left[N\left(w_{n}+i \Delta \omega\right)\right]\right\},
\end{aligned}
$$

where $\mathcal{P}_{r_{c}}^{S}=\left\langle S S\left|\hat{W}_{r} / \hat{W}_{c}\right| S S\right\rangle \ll 1$ is the probability of reaction in the well and

$$
N(\epsilon)=1-\left[1+\left(\epsilon / W_{e}\right)+\xi \sqrt{\epsilon / W_{e}}\right]^{-1}
$$

with $\xi=l_{e} \sqrt{W_{e} / D}$.

It is seen that in the limit of slow spin evolution, when $w_{p},\left|w_{n}+i \Delta \omega\right| \ll \xi^{2} W_{e}$, we get $N(\epsilon) \sim \sqrt{\epsilon}$ and formula (8.7) reduces to eq. (8.4), derived in the free diffusion model. Of great interest is also the opposite limit, in which the evolution kinetics is described by simple first order kinetic equations and $N(\epsilon) \sim\left(\epsilon / W_{e}\right)\left[1+\left(\epsilon / W_{e}\right)\right]^{-1}$. This kinetics shows itself in the typical analytical dependence of MFEs on relaxation rates and the splitting $\Delta \omega$.

\section{MFE for different relaxation mechanisms}

In our analysis (to reduce the number of parameters) we consider the limit of high reactivity at a contact, $\kappa_{S}^{d} / D \gg 1$, in which reaction/relaxation supermatrices $\hat{l}$ and $\hat{W}_{r}$ are independent of $\kappa_{S}$ :

$$
\hat{l} \approx d \hat{\overline{\mathcal{P}}}_{S}, \quad \hat{W}_{r} \approx w_{r} \hat{\overline{\mathcal{P}}}_{S} \text { with } \hat{\mathcal{\mathcal { P }}}_{S}=2 \hat{\mathcal{P}}_{S}-\hat{P}_{S S} .
$$
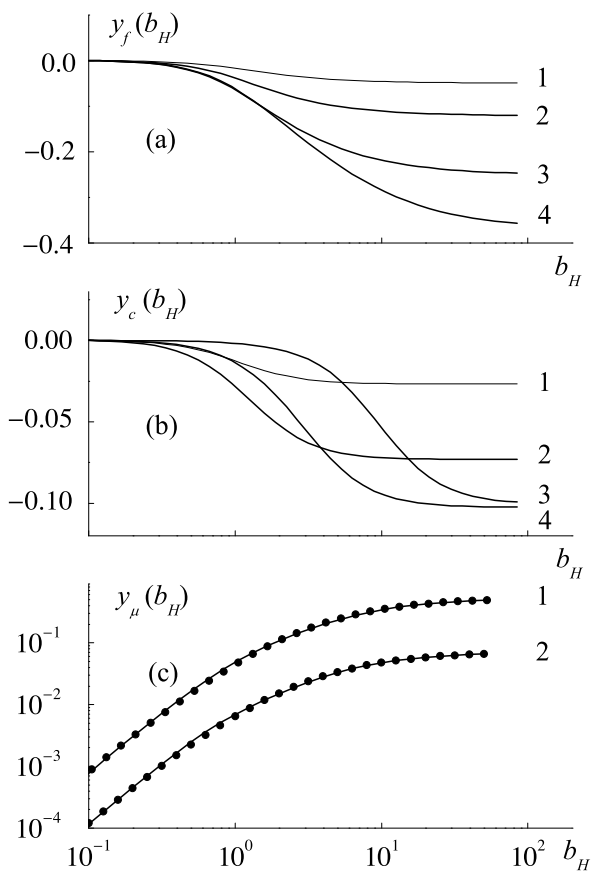

FIG. 2: Dependences $y_{\mu}\left(b_{H}\right)$ on $b_{H}=B / B_{H}$ for the HFI induced relaxation mechanism, calculated in free diffusion $(\mu=f)$ and cage $(\mu=c)$ models: for Lorenzian (Figs $2 \mathrm{a}$ and 2b) and non-Lorenzian (Fig. 2c) shapes of $\mathcal{J}_{H}(B)$. Fig. 2a shows $y_{f}\left(b_{H}\right)$ for different $\eta_{H}=d \sqrt{\bar{w}_{H} / D}: \eta_{H}=$ $0.1(1) ; 0.3(2) ; 1.0(3) ; 3.0(4)$. Fig. $2 \mathrm{~b}$ shows $y_{c}\left(b_{H}\right)$ for $r_{c}=w_{r} / W_{c}=0.5$ and different $\kappa_{H}=W_{c} / \bar{w}_{H}: \kappa_{H}=$ 5.0 (1); $1.0(2) ; 0.1$ (3); 0.01 (4). Fig. 2c shows arbitrarily scaled $y_{\mu}\left(b_{H}\right)$ for non-Lorenzian $\mathcal{J}_{H}(B)$ (Sec. VIII.C.1) with $\eta_{H}=10^{-4}$ for $\mu=f(1)$ and $r_{H}=1.0, \kappa_{H}=5.0$ for $\mu=c(2)$. Circles represent the prediction of formula $y_{\text {ex }}(B) \sim\left[B /\left(B_{0}+|B|\right)\right]^{2}$ with $B_{0}=2.35 B_{H}$, obtained by fitting $y_{\mu}\left(b_{H}\right)$.

Here $\hat{\mathcal{P}}_{S}$ is defined in eq. (5.5) and $w_{r}=D /\left(\kappa_{r} Z_{w}\right)$.

We also discuss in detail the special case of Lorenzian shape of functions $\mathcal{J}_{Q}(B)=\left(1+B^{2} / B_{Q}^{2}\right)^{-1}$, for which the relaxation rates are conveniently represented as

$$
w_{H}^{p}=\bar{w}_{H} /\left(1+b_{H}^{2}\right) \text { and } w_{A}^{p}=\bar{w}_{A}^{*} b_{A}^{2} /\left(1+b_{A}^{2}\right),
$$

where $b_{Q}=B / B_{Q}$ with $B_{Q}$ being the width of $\mathcal{J}_{Q}(B)$.

\section{HFI induced relaxation}

Specific features of dependences $y_{\mu}(B)$ for the HFIinduced relaxation mechanism are demonstrated in Fig. 2 for the free diffusion $(\mu=f)$ and diffusive cage $(\mu=c)$ models. In both models this mechanism leads to the de- 
creasing functions $y_{\mu}(B)$. The decrease results, evidently, from that of population relaxation rates as $B$ increased.

As expected from analytical expressions (8.4) and (8.7) these models predict similar dependence $y_{\mu}(B)$. In a wide region of values of the diffusion coefficient and reactivity the change of these parameters is found to lead mainly to the change of the MFE amplitude rather than to that of the shape of $y_{\mu}(B)$-dependence.

The shape is mostly determined by the mechanism of relaxation, or more accurately by the form of the correlation function $\mathcal{J}_{H}(B)$. For example, in the case of Lorenzian $\mathcal{J}_{H}(B)$ [see eq. [8.10)] the MFE shape is close to Lorenzian with the width of order of that for $\mathcal{J}_{H}(B)$.

In our short discussion we are not going to review all properties of the shape of $y_{\mu}(B)$. Majority of them can be described with formulas (8.4) and (8.7). More thoroughly we will only analyze the possibility of interpretation of the non-Lorenzian MFE shape $y_{e x}(B) \sim\left[B /\left(B_{0}+|B|\right)\right]^{2}$, observed in some experiments $\frac{7,15}{1.15}$

Note that for the non-Lorenzian function $\mathcal{J}_{H}(B)$ the MFEs $y_{\mu}(B)$ are non-Lorenzian as well. Just this property of the HFI induced relaxation mechanism allows one to describe the observed dependence $y_{e x}(B)$. The reaction yields $y_{f}(B)$ and $y_{c}(B)$, shown in in Fig.2c, are obtained for $\mathcal{J}_{H}^{\mu}(B), \quad(\mu=f, c)$, represented as sums of Lorenzian contributions $L o_{n}^{\mu}\left(b_{H}\right)=\left[1+b_{H}^{2} /\left(j_{n}^{\mu}\right)^{2}\right]^{-1}$, in which $j_{n}$ are the numerical coefficients: $\mathcal{J}_{H}(B) \sim$ $\sum_{n=1}^{6} a_{n}^{\mu} L o_{n}^{\mu}\left(b_{H}\right),(\mu=f, c)$, where $a_{i}^{\mu}$ are the weights of the Lorenzian contributions, taken the same both for the free diffusion and diffusive cage models: $a_{i}^{f}=a_{i}^{c}=1,(i=$ $1, \ldots, 6)$. The values of $j_{i}^{f}$ and $j_{i}^{c}$, conveniently represented as vectors $\mathbf{j}^{\mu}=\left(j_{1}^{\mu}, \ldots, j_{n}^{\mu}\right)$, are different in these models: $\mathbf{j}^{f}=(1,2,4,4,6,10)$ and $\mathbf{j}^{c}=(1,3,4,5,7.5,20)$. The chosen number of terms, $n=6$, seems to be the smallest of those, which are sufficient to get the yields quite close to $y_{e x}(B)$.

Thus calculated $y_{f}(B)$ and $y_{c}(B)$ agree with $y_{e x}(B)$ quite well in the wide region of $B$ values.

The obtained wide spectrum of widths, required in both models, is qualitatively consistent with the wide distribution of hopping rates (quite natural for disordered semiconductors), which determines the behavior of correlation functions, according to eq. (4.9).

\section{AZI induced relaxation}

Unlike the HFI induced relaxation the AZI induced one results in the increasing dependences $y_{\mu}(B)$ in both models of PP migration. As for the characteristic features of the shape of these dependences, they are not very similar to those for HFI induced relaxation. Typical dependences $y_{f}(B)$ and $y_{c}(B)$ are displayed in Figs. $3 \mathrm{a}$ and $3 \mathrm{~b}$ for Lorenzian shape of $\mathcal{J}_{A}(B)$ [see eq. (8.10)]

It is seen from Figs. 3a and $3 \mathrm{~b}$ that for AZI mechanism the widths of $y_{f}(B)$ and $y_{c}(B)$ are larger than those for HFI mechanism and, in general, are independent of the

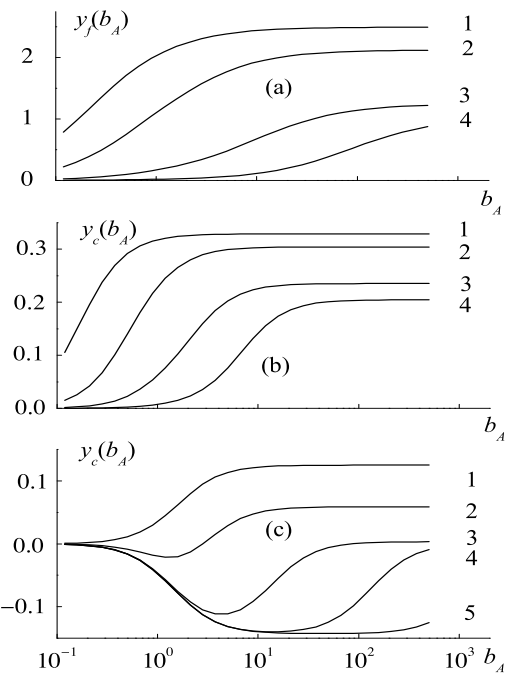

FIG. 3: The dependences $y_{\mu}\left(b_{A}\right)$ on $b_{A}=B / B_{A}$ for the AZI induced relaxation mechanism and for the Lorenzian shape of $\mathcal{J}_{A}(B)$, calculated in free diffusion $(\mu=f)$ and cage $(\mu=c)$ models (Figs. $3 \mathrm{a}$ and $3 \mathrm{~b}$ ), and $y_{c}\left(b_{A H}\right)$ for the HFI+AZI relaxation mechanism [assuming $\mathcal{J}_{H}(B)=\mathcal{J}_{A}(B)$ and $b_{A}=$ $b_{H}$ ] (Fig. 3c). Fig. 3a displays $y_{f}\left(b_{A}\right)$ for different $\eta_{A}=$ $d \sqrt{\bar{w}_{A}^{*} / D}: \quad \eta_{A}=0.02(1) ; 0.2(2) ; 2.0(3) ; 10.0$ (4). Fig. 3b displays $y_{c}\left(b_{H}\right)$ for $r_{c}=w_{r} / W_{c}=0.5$ and different $\kappa_{A}=$ $W_{c} / \bar{w}_{A}^{*}: \kappa_{A}=0.05(1) ; 0.5(2) ; 5(3) ; 50$ (4). Fig. 3c displays $y_{c}\left(b_{A}\right)$ for $r_{c}=1, \kappa_{H}=W_{c} / \bar{w}_{H}=1$, and different $\kappa_{A}=$ $W_{c} / \bar{w}_{A}^{*}: \kappa_{A}=1(1) ; 2.25(2) ; 4(3) ; 10^{2}(4) ; 10^{4}(5)$.

width $B_{A}$ of $\mathcal{J}_{A}(B)$. The reason of this independence consists in that, unlike the HFI induced relaxation, the AZI induced one results in dephasing, whose rate rapidly increases with $B: w_{A}^{n} \sim \bar{w}_{A} \sim B^{2}$ [see eq. (8.2)]. In such a case the width of $y_{\mu}(B)$ is mainly determined by the kinetic saturation of the MFEs observed for large dephasing rates $w_{A_{e, h}}^{n}$ [Sec. IV.A2 and eq. (8.1)]. In the free diffusion model the saturation occurs at $\left|k_{w}\right| d>1$, i.e. outside the region of validity of the fast diffusion limit (8.4) (Sec. VI.A2). As to the cage model, the saturation in this model is observed for $\|\hat{\Lambda}\| /\left\|\hat{W}_{c}\right\|>1$. Within the weak reactivity limit (8.7) this condition reduces to simple inequality $w_{n} / W_{e}>1$.

Most clearly the peculiarities of MFEs, resulting from AZI induced relaxation, manifest themselves in the limits of fast free diffusion and fast escaping from the cage $\left(w_{p, n} / W_{e} \ll 1\right)$. In these limits obtained formulas predict [see eqs. (8.4) and (8.7)] monotonically increasing behavior: $y_{f}(B) \sim|B|$ and $y_{c}(B) \sim B^{2}$ in the region of $B$ wider than that $\left(B \lesssim B_{A}\right)$ expected from eq. (8.10).

It is worth noting that the dependences close to $y(B) \sim|B|$ has been recently observed in a number of experiments: $\stackrel{13,15,21}{ }$ The proposed analysis allow for quite 
reasonable interpretation of this, at first sight, strange behavior.

\section{Superposition of HFI and AZI induced relaxation}

In the case of comparable contribution of HFI and AZI induced relaxation the free diffusion and diffusive cage models predict a large variety of MFE dependences $y_{\mu}(B),(\mu=f, c)$. In our brief analysis it is hardly possible to describe all types $y_{\mu}(B)$ behavior. In general, the superposition of HFI and AZI (HFI+AZI) induced relaxation mechanisms results in non-monotonic functions $y_{\mu}(B)$. Some representative examples of them, as applied to $y_{c}(B)$, are displayed in Fig. 3c. Analysis shows that the resulting shape of $y_{\mu}(B)$ dependence is essentially determined by the relative widths and relative weights (i.e. $\kappa_{H}^{-1}=\bar{w}_{H} / W_{c}$ and $\kappa_{A}^{-1}=\bar{w}_{A}^{*} / W_{c}$ ) of both mechanisms.

Naturally, as the weight of the AZI contribution increases $y_{\mu}(B)$ behavior changes from decreasing to increasing. Note that the change of the sign of the dependence $y_{\mu}(B)$ has recently observed in a number of experiments. The proposed theory can, in principle, be useful in interpretation of some of these observations.

In addition to this short discussion few comments are worth-wile, nevertheless, on the peculiar non-monotonic behavior of $y(B)$ functions at small $B$, sometime with the small maximum at $B=0$, which is found for some sets of parameters of models (see the curve 2 in Fig. 3c). The fact is that such a behavior has recently been observed in some semiconductor devices. ${ }^{16}$ In principle, the authors of this work assume that these non-monotonic dependences result from the exchange interaction between polarons at short distances. Our results show that some other mechanisms, for example the superposition of HFI and AZI induced relaxation, can lead to a similar $y(B)$ behavior.

\section{4. $\Delta g$-mechanism}

The conventional $\Delta g$-mechanism implies the MFE generation caused by quantum transitions between $S$ and $T$ states of the PP (4.17)- (4.19), which result from the spitting $\Delta \omega=(\Delta g) \beta B$ of Zeeman frequencies (see Sec. IV.B) .19 The difference of $g$-factors of $e$ - and $h$-polarons results from the spin-orbital coupling of electron spins of polarons in semiconductors,,$\frac{31}{2}$ i.e. the $\Delta g$-mechanism can be considered as one of manifestations of the this coupling in MFEs.

In our analysis of $\Delta g$-mechanism we neglect the effect of other (relaxation) mechanisms discussed above.

The manifestation of $\Delta g$-mechanism in many types of MFEs is analyzed in detail in a large number of works as applied to different chemical and physical processes. 19 The contribution of $\Delta g$-mechanism to the PP recombination yield $y_{\mu}(B)$ is also discussed in literature. Just this mechanism is believed to be responsible for $y_{\mu}(B) \sim \sqrt{B}$ behavior found in some semiconductors at large fields $B .13,15$ Moreover, in a number of works this behavior is used as a definition of the $\Delta g$-mechanism. Such a definition is somewhat misleading.

The fact is that $\Delta g$-mechanism, originally describing the effect of the term of the Hamiltonian $H$ (4.14) proportional to $\Delta \bar{g}$, predicts different $y_{\mu}(B)$ dependences, depending on the kinetics of relative motion of polarons:

a. Free diffusion model. In the case of free diffusion the dependence $y_{f}(B)$ can approximately be represented as $26,35 y_{f}(B) \sim \sqrt{\Delta \omega}\left(1+\xi_{f} \sqrt{\Delta \omega}\right)^{-1}$, where $\Delta \omega=(\Delta g) \beta B$ and $\xi_{f} \sim d / \sqrt{D}$. This formula shows for $d \sqrt{\Delta \omega / D} \ll 1$ we get the dependence $y_{f}(B) \sim \sqrt{\Delta \omega} \sim$ $\sqrt{B}$ (in some works considered as the manifestation $\Delta g$ mechanism), whereas in the opposite limit $d \sqrt{\Delta \omega / D}>1$ the dependence $y_{f}(B)$ saturates, i.e $y_{f}(B) \sim$ const.

b. Diffusive cage model. In the diffusive cage model we consider the case of deep well, when the effect of recapture of escaping particles is small. In the deep well limit the term $\sim \hat{k}_{0} \sim \sqrt{\hat{\Lambda}}$ in $\hat{\mathbf{P}}_{c}$ [eq. (6.31)] can be neglected so that $y_{c}(B)$ dependence (which becomes analytical) can approximated by ${ }^{34,36} y_{c}(B) \sim(\Delta \omega)^{2} /\left[1+\xi_{c}(\Delta \omega)^{2}\right]$, where $\xi_{c} \sim\left\|\hat{W}_{c}\right\|^{-2}$ [in the weak reactivity limit $\xi_{c} \sim$ $W_{e}^{-2}$, as it is seen from eq. (8.8)]. This means that in the cage model $\Delta g$-mechanism predicts rapidly increasing behavior of $y_{c}(B)$ at small $B<\left\|\hat{W}_{c}\right\| /(\bar{g} \beta): y_{c}(B) \sim B^{2}$, saturating at large $B>\left\|\hat{W}_{c}\right\| /(\bar{g} \beta): y_{c}(B) \sim$ const.

The simultaneous contributions of $\Delta g$-mechanism as well as HFI- and ASI-induced relaxation mechanisms can result in the additional specific features of the MFE shape $y_{\mu}(B)$. We are not going to discuss them in this work, but only mention that typically $\Delta g$-mechanism strongly contributes at large $B$, at which contributions of both other mechanisms are nearly independent of $B$. In this case the change of MFE shape caused by $\Delta g$-mechanism can easily be identified and described if needed.

\section{CONCLUDING REMARKS}

In this work PP recombination mechanisms of MFEs in disordered semiconductors are analyzed in detail. The magnetic field dependent PP recombination yield is discussed as the most well known example of the MFE observable. The hopping migration of polarons is assumed to result not only in the spatial evolution of polarons, but also in fluctuations of the HFI and AZI of polarons, thus leading to HFI and AZI induced spin relaxation. In our work we have considered the manifestation of these two mechanism of MFEs as well as $\Delta g$-mechanism.

Simple analytical formulas are derived for the recombination yield $Y(B)$ in two models of polaron migration: the free diffusion model and the model of diffusion in the well, or diffusive cage model. Analysis demonstrates that specific features of polaron migration shows itself in the shape of $Y(B)$. Most important typical properties of 
$Y(B)$-dependence for all three MFE mechanisms and in both models of migration are discussed in detail.

Concluding this discussion we would like to point out two important points:

1) Above-obtained results show that the width $B_{r}$ of the relaxation induced MFE (PP recombination yield) $y_{\mu}(B)$ is determined by the hopping rate: $B_{r} \sim w_{0} /(g \beta)$, i.e. is much larger than typical HFI: $B_{r} \gg \sqrt{\left\langle B_{\nu}^{2}\right\rangle}$.

2) The proposed theory is applied to analysis of the magnetic field dependent yield of recombination of $e-h$ PPs, i.e. polarons with charges of opposite sign. It is clear, however, that obtained results are quite applicable to description of recombination of $e-e$ PPs or $h-h$ PPs as well. Just this kind of processes is recently considered as a mechanism of MFEs in organic semiconductors. ${ }^{37}$

3) This work concerns the analysis of recombination of polarons, i.e. particles with the electron spin $1 / 2$. The obtained matrix formulas are, however, quite general and can be applied to describing similar processes with participation of particles with higher spins, for example, triplet exciton-polaron quenching, 18,19,21 triplettriplet annihilation, $\frac{18,19}{19}$ etc. The analysis of these processes is a subject of future works.

Acknowledgements. The work was partially supported by the Russian Foundation for Basic Research.
1 R. Johnson, R. Merrifield, P. Avakian, and R R. Flippen, Phys. Rev. Lett. 19, 285 (1967).

2 R. Groff, R. Merrifield, A. Suna, and P. Avakian, Phys. Rev. Lett. 29, 429 (1972).

3 E. F. Frankevich, A. Zakhidov, K. Yoshino, Y. Maruyama, and K. Yakushi, Phys. Rev. B 53, 4498 (1996).

${ }^{4}$ V. N. Prigodin, N. P Raju, K. I. Pokhodnya, J. S. Miller, and A. J. Epstein, Adv. Mater. 14, 1230 (2002).

5 M. Wohlgenannt, C. Yang, and Z. V. Vardeny, Phys. Rev. B 66, 241201(R) (2002).

6 Z. Xiong, D. Wu, Z. V. Vardeny, and J. Shi, Nature (London) 427, 821 (2004).

7 Ö. Mermer, G. Veeraraghavan, T. L. Francis, Y. Sheng, D. T. Nguyen, M. Wohlgenannt, A. Köhler, M. K. Al-Suti, and M. S. Khan, Phys. Rev. B 72, 205202 (2005).

8 V. N. Prigodin, J. D. Bergeson, D. M. Lincoln, and A. J. Epstein, Synth. Met. 156, 757 (2006).

9 Y. Sheng, D. T. Nguyen, G. Veeraraghavan, Ö. Mermer, M. Wohlgenannt, S.Qiu, and U. Scherf, Phys. Rev. B 74, 045213 (2006).

10 T. D. Nguyen, Y. Sheng, J. Rybicki, and M. Wohlgenannt, Phys. Rev. B 77, 235209 (2008).

11 C. G. Yang, E. Ehrenfreund, F. Wang, T. Drori, and Z. V. Vardeny, Phys. Rev. B 78, 205312 (2008).

12 J. D. Bergeson, V. N. Prigodin, D. M. Lincoln, and A. J. Epstein, Phys. Rev. Lett. 100, 067201 (2008).

13 F. J. Wang, H. Bässler, and Z. Valy Vardeny, Phys. Rev. Lett. 101, 236805 (2008).

14 P. A. Bobbert, W. Wagemans, F. W. A. van Oost, B. Koopmans, and M. Wohlgenannt, Phys. Rev. Lett. 102, 156604 (2009).

15 S. Majumdar, H. S. Majumdar, H. Aarnio, D. Vanderzande, R. Laiho, and R Österbacka, Phys. Rev. B 79, 201202(R) (2009).

16 T. D. Nguyen, B. R. Gautam, E. Ehrenfreund, and Z. V. Vardeny, Phys. Rev. Lett. 105, 166804 (2010).

17 P. A. Bobbert, T. D. Nguyen, W. Wagemans, F. W. A. van Oost, B. Koopmans, and M. Wohlgenannt, Synth. Met. 160, 223 (2010).
18 C. E. Swenbwerg and N. E. Geacintov, in Organic Molecular Photophysics, v. 1, edited by J. B. Birks (Wiley, NY, 1973).

19 U. E. Steiner and T. Ulrich, Chem. Rev. 89, 51 (1989).

20 V. A. Dediu, L. E. Hueso, I. Bergenti, and C. Taliani, Nature Mater. 8, 707 (2009).

21 P. Desai, P. Shakya, T. Kreouzis, W. P. Gillin, N. A. Morley, and M. R. J. Gibbs, Phys. Rev. B 75, 094423 (2007).

22 P. Desai, P. Shakya, T. Kreouzis, and W. P. Gillin, J. Appl. Phys. 103, 043706 (2008).

23 S. Zhang, J. Song, and W. P. Gillin, J. Appl. Phys. 106, 043511 (2009).

24 J. H. Freed and J. B. Pedersen, Adv. Magn. Reson. 8, 1 (1976).

25 A. I. Shushin, Chem. Phys. 144, 201 (1990); 144, 223 (1990)

26 A. I. Shushin, Chem. Phys. Lett. 118, 197 (1985) J. Chem. Phys. 95, 3657 (1991).

27 A. I. Shushin, J. Chem. Phys. 97, 1954 (1992); Phys. Rev. E 62, 4688 (2000).

28 A. Miller and E. Abrahams, Phys. Rev. 120, 745 (1960).

29 J. J. M. van der Holst, F. W. A. van Oost, R. Coehoorn, and P. A. Bobbert, Phys. Rev. B 80, 235202 (2009).

30 A. Abragam, The principles of nuclear magnetism (Clarendon Press, Oxford, 1961).

31 A. Carrington and A. D. McLachlan, Introduction to magnetic resonance (Harper\&Row Publishers, New York, 1967).

32 K. Schulten and P. G. Wolynes, J. Chem. Phys. 68, 3292 (1978).

33 A. I. Shushin, J. Chem. Phys. 116, 9792 (2002).

34 A. I. Shushin, Mol. Phys. 58, 101 (1986).

35 A. I. Shushin, Chem. Phys. Lett. 85, 562 (1982).

36 A. I. Shushin, J. B. Pedersen, and L. I. Lolle, Chem. Phys. 188, 1 (1994).

37 P. A. Bobbert, T. D. Nguyen, F. W. A. van Oost, B. Koopmans, and M. Wohlgenannt, Phys. Rev. Lett. 99, 216801 (2007). 